\title{
Aspherical manifolds that cannot be triangulated
}

\author{
MiCHAEL W DAVIS \\ JIM FOWLER \\ JEAN-FRANÇOIS LAFONT
}

\begin{abstract}
By a result of Manolescu [14] there are topological closed $n$-manifolds that cannot be triangulated for each $n \geq 5$. We show here that for $n \geq 6$ we can choose such manifolds to be aspherical.
\end{abstract}

57Q15; 20F65, 57Q25, 57R58

\section{Introduction}

Although Kirby and Siebenmann [13] showed that there are manifolds which do not admit PL structures, the possibility remained that all manifolds could be triangulated. In the late seventies Galewski and Stern [10] constructed a closed 5-manifold $M^{5}$ so that every $n$-manifold, with $n \geq 5$, can be triangulated if and only if $M^{5}$ can be triangulated. Moreover, $M^{5}$ admits a triangulation if and only if the Rokhlin $\mu-$ invariant homomorphism, $\mu: \theta_{3}^{H} \rightarrow \mathbb{Z} / 2$, is split. In 2013 Manolescu [14] showed that the $\mu$-homomorphism does not split. Consequently, there exist Galewski-Stern manifolds $M^{n}$ that are not triangulable for each $n \geq 5$.

In 1982 Freedman [8] proved that there exists a topological 4-manifold with even intersection form of signature 8. It followed from later work of Casson that such 4-manifolds could not be triangulated. In 1991 Davis and Januszkiewicz [5] applied Gromov's hyperbolization procedure to Freedman's $E_{8}$-manifold to show that there exist closed aspherical 4-manifolds that cannot be triangulated.

In this paper we apply hyperbolization techniques to the Galewski-Stern manifolds to show that there exist closed aspherical $n$-manifolds that cannot be triangulated for each $n \geq 6$. The question remains open in dimension 5 .

Any 3-dimensional homology sphere $H^{3}$ bounds a PL 4-manifold $W^{4}$ with vanishing first and second Stiefel-Whitney classes. The intersection form of $W^{4}$ is then unimodular and even; so, its signature $\sigma\left(W^{4}\right)$ is divisible by 8 . If $H^{3}$ bounds an 
acyclic 4-manifold (eg if $H^{3}=S^{3}$ ), then, by Rokhlin's Theorem, $\sigma\left(W^{4}\right)$ is divisible by 16 . So, one defines the $\mu$-invariant of $H^{3}$ by

$$
\mu\left(H^{3}\right)=\frac{\sigma\left(W^{4}\right)}{8} \bmod 2 .
$$

Let $\theta_{3}^{H}$ be the abelian group (under connected sum) of homology cobordism classes of oriented PL homology 3 -spheres. The $\mu$-invariant defines a homomorphism $\mu: \theta_{3}^{H} \rightarrow \mathbb{Z} / 2$. (For background about this material, see Saveliev [17].)

In [13] Kirby and Siebenmann proved that for any topological manifold $M^{n}$ there is an obstruction $\Delta \in H^{4}\left(M^{n} ; \mathbb{Z} / 2\right)$ which, for $n \geq 5$, vanishes if and only if $M^{n}$ admits a PL structure. An important point here is that the Kirby-Siebenmann obstruction can be defined for any polyhedral homology manifold $M^{n}$ as follows. First, there is obstruction in $H^{4}\left(M^{n} ; \theta_{3}^{H}\right)$ to finding an acyclic resolution of $M$ by a PL manifold. This is the class of the cocycle that associates to each 4-dimensional "dual cell" in $M^{n}$ the class of its boundary in $\theta_{3}^{H}$. The Kirby-Siebenmann obstruction $\Delta$ is the image of this element from $H^{4}\left(M^{n} ; \theta_{3}^{H}\right)$ under the coefficient homomorphism $\mu: \theta_{3}^{H} \rightarrow \mathbb{Z} / 2$.

After the proof by Edwards and Cannon of the double suspension theorem, it seemed possible that every topological manifold could still be homeomorphic to some simplicial complex even if it did not have a PL structure. Galewski and Stern [11; 9] and independently Matumoto [15] proved that the following statements are equivalent.

(a) Every manifold of dimension $\geq 5$ can be triangulated.

(b) There exists a homology 3-sphere $H^{3}$ with $\mu\left(H^{3}\right)=1$ and $\left[H^{3}\right]$ of order 2 in $\theta_{3}^{H}$.

Galewski and Stern also showed that, for $n \geq 5$, the obstruction for a manifold to have a simplicial triangulation was the Bockstein of its Kirby-Siebenmann obstruction, $\beta(\Delta) \in H^{5}\left(M^{n} ; \operatorname{Ker} \mu\right)$, where $\beta: H^{4}\left(M^{n} ; \mathbb{Z} / 2\right) \rightarrow H^{5}\left(M^{n} ; \operatorname{Ker} \mu\right)$ denotes the Bockstein homomorphism associated to the short exact sequence of coefficients

$$
0 \longrightarrow \operatorname{Ker} \mu \longrightarrow \theta_{3}^{H} \stackrel{\mu}{\longrightarrow} \mathbb{Z} / 2 \longrightarrow 0 .
$$

In fact, as was shown by Galewski and Stern [10], one can focus instead on a simpler Bockstein associated to the short exact sequence of coefficients

$$
0 \longrightarrow \mathbb{Z} / 2 \stackrel{\times 2}{\longrightarrow} \mathbb{Z} / 4 \longrightarrow \mathbb{Z} / 2 \longrightarrow 0 .
$$

It is well-known that the Bockstein associated to (1) is the first Steenrod square, $\mathrm{Sq}^{1}$. So, henceforth we will use $\mathrm{Sq}^{1}$ instead of a more general Bockstein $\beta$. The reduction to the case of $\mathrm{Sq}^{1}$ goes as follows. Suppose $N^{n}$ is a manifold with $\operatorname{Sq}^{1}(\Delta) \neq 0$. By [10, Theorem 2.1], if one such $N^{n}$ can be triangulated, then every manifold of 
dimension $\geq 5$ can be triangulated (this is statement (a) above). In [10] Galewski and Stern also constructed $n$-manifolds, for each $n \geq 5$, with $\operatorname{Sq}^{1}(\Delta) \neq 0$.

Manolescu [14, Corollary 1.2] recently established that homology 3-spheres as in (b) do not exist. It follows that any manifold with $\operatorname{Sq}^{1}(\Delta) \neq 0$ is not homeomorphic to a simplicial complex. So, Galewski-Stern manifolds cannot be triangulated.

By work of Freedman and Casson nontriangulable manifolds exist in dimension 4; cf Akbulut and McCarthy [1]. First, Freedman [8] showed that any homology 3sphere bounds a contractible (topological) 4-manifold. One defines the $E_{8}$-homology manifold $X^{4}$ as follows. Start with the plumbing $Q\left(E_{8}\right)$ defined by the $E_{8}$ diagram. It is a smooth, parallelizable 4-manifold with boundary; its boundary being Poincaré's homology 3-sphere, $H^{3}$. The signature of $Q\left(E_{8}\right)$ is $8 . X^{4}$ is defined to be the union of this plumbing with $c\left(H^{3}\right)$ (the cone on $H^{3}$ ). It is a polyhedral homology 4manifold with one nonmanifold point. By Freedman [8] we can topologically "resolve the singularity" of $X^{4}$ by replacing $c\left(H^{3}\right)$ with a contractible 4-manifold bounded by $H^{3}$ to obtain a topological 4-manifold $M^{4}$ with nontrivial Kirby-Siebenmann invariant. In fact, any triangulation of a 4-manifold is automatically PL. (Proof: The link of a simplex of dimension $>0$ is a homology sphere of dimension $\leq 2$, hence, a sphere; similarly, the link of a vertex is a simply connected homology 3-sphere, hence, by the Poincaré Conjecture, a 3-sphere.) So, if a 4-dimensional manifold does not admit a PL structure, then it cannot be triangulated.

A variation of this idea was used in Davis and Januszkiewicz [5] to produce an aspherical 4-manifold that cannot be triangulated. Start with a triangulation of $X^{4}$. Apply the "hyperbolization" technique of Gromov [12] to $X^{4}$ to get $h\left(X^{4}\right)$. It is a locally CAT(0), polyhedral homology manifold with one nonmanifold point, the link of which is $H^{3}$. Resolve the conical singularity to obtain a closed aspherical topological manifold $N^{4}$ with $\Delta\left(N^{4}\right) \neq 0$. By the previous argument, $N^{4}$ cannot be triangulated. (However, $N^{4} \times S^{1}$ can be triangulated since, by the double suspension theorem, it is homeomorphic to the triangulated manifold $h\left(X^{4}\right) \times S^{1}$.)

The idea of this paper is to apply hyperbolization techniques to the Galewski-Stern manifolds to obtain aspherical manifolds $N^{n}$ that cannot be triangulated. We do not know how to make our techniques work in dimension 5; however, they do work in any dimension $\geq 6$. So, we get the following.

Main Theorem For each $n \geq 6$ there is a closed aspherical manifold $N^{n}$ that cannot be triangulated.

Acknowledgements Our thanks go to Ron Stern and to the referee for helpful comments. 


\section{The construction}

The Galewski-Stern 5-manifold We recall the Galewski-Stern construction in [10] of a 5-manifold, $N^{5}$, now known to be nontriangulable. Start with $X^{4} \times I$, where $X^{4}$ is the $E_{8}$-homology manifold. Attach an orientation-reversing 1-handle, $D^{3} \times I$, connecting the two copies of $c\left(H^{3}\right)$ along their boundary. The two copies of $Q\left(E_{8}\right)$ become the boundary connected sum $Q\left(E_{8}\right) \#_{b} Q\left(E_{8}\right)$; it is a 4-manifold with boundary, the boundary being $H^{3} \# H^{3}$ (not $H^{3} \#\left(-H^{3}\right)$ ). Consider $c\left(H^{3}\right) \cup$ $\left(D^{3} \times I\right) \cup c\left(H^{3}\right)$. It is a contractible polyhedral homology 4-manifold; its boundary is $H^{3} \# H^{3}$. Fill in the boundary with $c\left(H^{3} \# H^{3}\right)$ to obtain a polyhedral homology manifold $T$ with the homology of $S^{4}$ (ie a "generalized homology 4-sphere"). Next fill in $T$ with $c(T)$. The result is the polyhedral 5-manifold with boundary

$$
P^{5}:=\left(X^{4} \times I\right) \cup c(T) .
$$

Roughly speaking, after ignoring the differences between homology spheres and spheres, we have attached an orientation-reversing 1-handle $D^{4} \times I$ to $X^{4} \times I$ (the 1-handle is actually $c(T))$. So, $P^{5}$ is a nonorientable, polyhedral homology 5 -manifold with boundary; it is homotopy equivalent to $S^{1} \vee X^{4}$. The boundary of $P^{5}$ is $c\left(H^{3} \# H^{3}\right) \cup$ $Q\left(E_{8}\right) \#_{b} Q\left(E_{8}\right)$; so, $\partial P^{5}$ contains a single nonmanifold point (the cone point of $\left.c\left(H^{3} \# H^{3}\right)\right)$. By Edwards' polyhedral-topological manifold characterization theorem [7, page 119], the interior of $P^{5}$ is a topological manifold. Its Kirby-Siebenmann invariant, $\Delta\left(P^{5}\right)$, is the image of $\left[X^{4}\right]$ in $H^{4}\left(P^{5} ; \mathbb{Z} / 2\right)$. Thus, $P^{5}$ is polyhedral homology manifold with boundary and its interior is a topological manifold which does not admit a PL structure. Since the Kirby-Siebenmann obstruction of $\partial P$ is $0, \Delta(P)$ is the image of a (unique) class $\Delta(P, \partial P) \in H^{4}(P, \partial P ; \mathbb{Z} / 2)$; moreover, one sees that the image $\operatorname{Sq}^{1}(\Delta(P, \partial P))$ under $\mathrm{Sq}^{1}$ is the nonzero class in $H^{5}(P, \partial P ; \mathbb{Z} / 2)$.

When it comes to applying hyperbolization, it is at this point where the GalewskiStern construction becomes problematic. Galewski and Stern get rid of the singular point of $\partial P^{5}$ as follows: (1) attach an external collar $\partial P \times[0,1]$ to $P,(2)$ find a PL manifold $V^{4}$ embedded in $\partial P \times(0,1)$, (3) define $U$ to be the part of the external collar between $\partial P \times 0$ and $V^{4}$, (4) argue that $V^{4}$ bounds a PL 5-manifold $W$ (necessarily nonorientable) and finally, (5) glue in $W$ to get the desired manifold,

$$
N^{5}:=P \cup U \cup W .
$$

Galewski-Stern manifolds of dimension $>\mathbf{5}$ In dimensions $>5$ there are versions of these manifolds to which it is easier to apply hyperbolization. To fix ideas, suppose the dimension is 6 . Let $P^{\prime}:=P^{5} \times S^{1}$, where $P^{5}$ is the polyhedral homology 5-manifold with boundary which was constructed previously. Then $\Delta\left(P^{\prime}\right)$ is the nontrivial element 
in $H^{4}(P ; \mathbb{Z} / 2) \otimes H^{0}\left(S^{1} ; \mathbb{Z} / 2\right)$, a summand of $H^{4}\left(P^{\prime} ; \mathbb{Z} / 2\right)$. It is the image of the unique nontrivial element $\Delta\left(P^{\prime}, \partial P^{\prime}\right)$ of $H^{4}(P, \partial P ; \mathbb{Z} / 2) \otimes H^{0}\left(S^{1} ; \mathbb{Z} / 2\right)$. Its image under $\mathrm{Sq}^{1}$ is denoted $\operatorname{Sq}^{1}\left(\Delta\left(P^{\prime}, \partial P^{\prime}\right)\right) \in H^{5}(P, \partial P ; \mathbb{Z} / 2) \otimes H^{0}\left(S^{1} ; \mathbb{Z} / 2\right) \cong \mathbb{Z} / 2$. By Edwards' Theorem, $\partial P^{\prime}$ is a topological 5-manifold. Since $\Delta\left(\partial P^{\prime}\right)$ is zero, $\partial P^{\prime}$ is actually homeomorphic to a PL 5-manifold $V^{\prime}$. It is easy to see that $V^{\prime}$ bounds a PL 6-manifold $W^{\prime}$. Put

$$
N^{\prime}:=P^{\prime} \cup U \cup W^{\prime},
$$

where $U$ is the mapping cylinder of a homeomorphism between $\partial P^{\prime}$ and $V^{\prime}=\partial W^{\prime}$. Since $\Delta\left(N^{\prime}\right)$ restricts to $\Delta\left(P^{\prime}\right)$, we have $\Delta\left(N^{\prime}\right) \neq 0$ and one sees as before that $\Delta\left(N^{\prime}\right)$ is the image of

$$
\Delta\left(P^{\prime}, \partial P^{\prime}\right) \in H^{4}\left(P^{\prime}, \partial P^{\prime} ; \mathbb{Z} / 2\right) \cong H^{4}\left(N^{\prime}, U \cup W^{\prime} ; \mathbb{Z} / 2\right) .
$$

By Wu's formula, $\operatorname{Sq}^{1}\left(\Delta\left(P^{\prime}, \partial P^{\prime}\right)\right)=w_{1}\left(P^{\prime}\right) \cup \Delta\left(P^{\prime}, \partial P^{\prime}\right) \neq 0$. (This argument is from the final paragraph of Galewski and Stern [10].) Hence, $\operatorname{Sq}^{1}\left(\Delta\left(N^{\prime}\right)\right)$ is the nonzero image of $\mathrm{Sq}^{1}\left(\Delta\left(P^{\prime}, \partial P^{\prime}\right)\right)$.

Hyperbolization A hyperbolization technique of Gromov [12, pages 114-117] is explained in Davis and Januszkiewicz [5]: given a simplicial complex $K$, one can construct a new space $h(K)$ and a map $f: h(K) \rightarrow K$ with the following properties.

(a) The space $h(K)$ is a locally CAT(0) cubical complex; in particular, it is aspherical.

(b) The inverse image in $h(K)$ of any simplex of $K$ is a "hyperbolized simplex." So, the inverse image of each vertex in $K$ is a point in $h(K)$.

(c) The map $f: h(K) \rightarrow K$ induces a split injection on cohomology; cf Davis and Januszkiewicz [5, page 355].

(d) Hyperbolization preserves local structure: for any simplex $\sigma$ in $K$ the link of $f^{-1}(\sigma)$ is isomorphic to a subdivision of the link of $\sigma$ in $K$; $\mathrm{cf}$ [5, page 356]. So, if $K$ is a polyhedral homology manifold, then so is $h(K)$.

(e) If $K$ is a polyhedral homology manifold, then $f: h(K) \rightarrow K$ pulls back the Stiefel-Whitney classes of $K$ to those of $h(K)$.

In Davis, Januszkiewicz and Weinberger [6] the above version of hyperbolization is used to define a "relative hyperbolization procedure" (an idea also due to Gromov [12, page 117]). Given $(K, \partial K)$, a triangulated manifold with boundary, form $K \cup c(\partial K)$ and then define $H(K, \partial K)$ to be the complement of an open neighborhood of the cone point in $h(K \cup c(\partial K))$. Then $H(K, \partial K)$ is a manifold with boundary; its boundary is homeomorphic to $\partial K$. The main result of [6] is that if each component 
of $\partial K$ is aspherical, then so is $H(K, \partial K)$; moreover, the inclusion $\partial K \rightarrow H(K, \partial K)$ induces an injection on fundamental groups for each component of $\partial K$. In other words, if an aspherical manifold bounds a triangulable manifold, then it bounds a triangulable aspherical manifold.

Proof of the Main Theorem Our nontriangulable 6-manifold $N^{6}$ will be the union of three pieces, $N^{6}=P_{1} \cup U \cup P_{2}$, where $P_{1}$ and $P_{2}$ are triangulable, aspherical 6-manifolds with boundary, and where $U$ is the mapping cylinder of a homeomorphism $\partial P_{1} \rightarrow \partial P_{2} . P_{1}$ will be defined via hyperbolization and $P_{2}$ via relative hyperbolization. Put $P_{1}:=h\left(P^{\prime}\right)$, where $P^{\prime}=P^{5} \times S^{1}$ is the simplicially triangulated 6-manifold with boundary discussed above. Then $P_{1}$ is a topological 6-manifold with boundary and $\partial P_{1}=h\left(\partial P^{\prime}\right)$ is homeomorphic to a PL 5-manifold $V$. (Note the PL structure on $V$ is incompatible with the triangulation of $\partial P_{1}$ as a subcomplex of $P_{1}$.) Let $U$ be the mapping cylinder of a homeomorphism $h\left(\partial P^{\prime}\right) \rightarrow V$. Let $W$ be a PL manifold bounded by $V$. Equip $W$ with a PL triangulation. Applying relative hyperbolization, we get an aspherical 6-manifold with boundary $P_{2}:=H(W, V)$; its boundary being $V$. Then $N^{6}=P_{1} \cup U \cup P_{2}$ is aspherical. By properties (c) and (d) of hyperbolization, $\Delta\left(P_{1}, \partial P_{1}\right)=f^{*}\left(\Delta\left(P^{\prime}, \partial P^{\prime}\right)\right)$. So, $\operatorname{Sq}^{1}\left(\Delta\left(P_{1}, \partial P_{1}\right)\right)=f^{*}\left(\operatorname{Sq}^{1}\left(\Delta\left(P^{\prime}, \partial P^{\prime}\right)\right)\right)$. Consequently, $\Delta\left(P_{1}, \partial P_{1}\right)$ and $\operatorname{Sq}^{1}\left(\Delta\left(P_{1}, \partial P_{1}\right)\right)$ are both nonzero. Since $P_{2}$ is a $\mathrm{PL}$ manifold, its obstructions vanish. As before, it follows that $\Delta\left(N^{6}\right)$ and $\operatorname{Sq}^{1}\left(\Delta\left(N^{6}\right)\right)$ are both nonzero.

\section{Remarks}

Dimension 5 What is the situation in dimension 5? As explained in the Introduction, any polyhedral homology 4-manifold, $M^{4}$, can be resolved to a topological manifold $M_{\text {res }}^{4}$. When $P^{5}$ is defined by (2), $\left(\partial P^{5}\right)_{\text {res }}$ does not support a PL structure (although $\left.\Delta\left(\left(\partial P^{5}\right)_{\text {res }}\right)=0\right)$. However, one can vary the definition of $P^{5}$ so that $\left(\partial P^{5}\right)_{\text {res }}$ becomes homeomorphic to a PL 4-manifold. For example, we could arrange for $\left(\partial P^{5}\right)_{\text {res }}$ to be the $\mathrm{K} 3$ surface. To complete our construction we need $\left(h\left(\partial P^{5}\right)\right)_{\text {res }}$, the resolution of the hyperbolization, to be PL. If this could be achieved, we could finish by finding a PL 5-manifold bounded by $\left(h\left(\partial P^{5}\right)\right)_{\text {res }}$ and then applying relative hyperbolization as before.

Word hyperbolicity We will show below that by using the strict hyperbolization technique of Charney and Davis [4], one can arrange for nontriangulable aspherical manifolds of dimension $\geq 6$ to have word hyperbolic fundamental groups. So, in this paragraph $h(K)$ is the strict hyperbolization functor of [4] and $H(K, \partial K)$ is the corresponding strict relative hyperbolization procedure. We take notation from the 
proof of the Main Theorem. Then $P_{1}=h\left(P^{\prime}\right)$ is locally CAT $(-1)$ and $\partial P_{1}=h\left(\partial P^{\prime}\right)$ is a totally geodesic subspace. Hence, $\pi_{1}\left(P_{1}\right)$ is word hyperbolic and $\pi_{1}\left(\partial P_{1}\right)$ is a quasiconvex subgroup. Belegradek [3, Theorem 1.1] proved that the fundamental group of $H(K, \partial K)$ is relatively hyperbolic with peripheral subgroup $\pi_{1}(\partial K)$. So, for $P_{2}=H\left(W, \partial P_{1}\right)$, we have that $\pi_{1}\left(P_{2}\right)$ is relatively hyperbolic and its peripheral subgroup $\pi_{1}\left(\partial P_{1}\right)$ is word hyperbolic. By Osin [16, Corollary 2.41] this implies that $\pi_{1}\left(P_{2}\right)$ is word hyperbolic. Since $\pi_{1}\left(P_{2}\right)$ is both relatively hyperbolic and word hyperbolic, it is a result of Bowditch that the peripheral subgroup $\pi_{1}\left(\partial P_{1}\right)$ is quasiconvex and malnormal in $\pi_{1}\left(P_{2}\right)$. Finally, Alibegović [2, Theorem 2.3] has shown that under the additional condition of "tightness" one can show that a graph of word hyperbolic groups is word hyperbolic. (Tightness is implied by the malnormality of the peripheral subgroup.) In the case at hand, the inclusions $\pi_{1}\left(\partial P_{1}\right) \hookrightarrow \pi_{1}\left(P_{1}\right)$ and $\pi_{1}\left(\partial P_{1}\right) \hookrightarrow \pi_{1}\left(P_{2}\right)$ are tight. Hence, the amalgamated product,

$$
\pi_{1}\left(N^{6}\right)=\pi_{1}\left(P_{1}\right) *_{\pi_{1}\left(\partial P_{1}\right)} \pi_{1}\left(P_{2}\right)
$$

is word hyperbolic.

Orientability The Galewski-Stern 5-manifold is nonorientable. (In fact, Siebenmann [18] showed that every orientable 5-manifold can be triangulated.) Since our construction of a nontriangulable aspherical manifold $N^{6}$ starts from $P^{5} \times S^{1}$, the manifold $N^{6}$ is also nonorientable (by property (e) of hyperbolization). The question arises: Do orientable examples exist? The answer is yes. To construct one, start from the nonorientable $S^{1}$-bundle over $P$ instead of $P \times S^{1}$, where $w_{1}$ of the associated vector bundle is $w_{1}(P)$. The total space $E$ of the $S^{1}$-bundle is then a 6 -dimensional, orientable, polyhedral homology manifold with boundary. The restriction of the $S^{1}-$ bundle to $\partial P^{5}$ is orientable so $\partial E=\partial P^{5} \times S^{1}$ remains the same. As before, we get $N^{6}=P_{1} \cup U \cup P_{2}$ where $P_{1}=h(E), V$ is a PL manifold homeomorphic to $\partial P_{1}, W$ is a PL manifold bounded by $V, P_{2}=H(W, V)$ and $U$ is the mapping cylinder of a homeomorphism $\partial P_{2} \rightarrow \partial P_{1}$.

Bounding nontriangulable manifolds By using relative hyperbolization, Davis, Januszkiewicz and Weinberger [6] proved that if an aspherical manifold bounds a triangulable manifold, then it bounds an aspherical one. Can we omit the word "triangulable?" In other words, if an aspherical topological manifold $\mathrm{M}$ bounds, does it bound an aspherical manifold? If $\mathrm{M}$ does not support a triangulation, then it cannot bound a triangulated manifold, and one cannot use relative hyperbolization directly. The specific examples of nontriangulable aspherical manifolds constructed in this paper are boundaries, and a similar construction can be applied to produce aspherical manifolds that they bound. However, the general question remains open. 


\section{References}

[1] S Akbulut, JD McCarthy, Casson's invariant for oriented homology 3-spheres, Mathematical Notes 36, Princeton Univ. Press (1990) MR1030042

[2] E Alibegović, A combination theorem for relatively hyperbolic groups, Bull. London Math. Soc. 37 (2005) 459-466 MR2131400

[3] I Belegradek, Aspherical manifolds with relatively hyperbolic fundamental groups, Geom. Dedicata 129 (2007) 119-144 MR2353987

[4] R M Charney, M W Davis, Strict hyperbolization, Topology 34 (1995) 329-350 MR1318879

[5] M W Davis, T Januszkiewicz, Hyperbolization of polyhedra, J. Differential Geom. 34 (1991) 347-388 MR1131435

[6] M W Davis, T Januszkiewicz, S Weinberger, Relative hyperbolization and aspherical bordisms: An addendum to "Hyperbolization of polyhedra", J. Differential Geom. 58 (2001) 535-541 MR1906785

[7] R D Edwards, The topology of manifolds and cell-like maps, from: "Proceedings of the International Congress of Mathematicians", (O Lehto, editor), Acad. Sci. Fennica, Helsinki (1980) 111-127 MR562601

[8] M H Freedman, The topology of four-dimensional manifolds, J. Differential Geom. 17 (1982) 357-453 MR679066

[9] D E Galewski, R J Stern, Simplicial triangulations of topological manifolds, from: “Algebraic and geometric topology, Part 2", (R J Milgram, editor), Proc. Sympos. Pure Math. 32, Amer. Math. Soc. (1978) 7-12 MR520518

[10] D E Galewski, R J Stern, A universal 5-manifold with respect to simplicial triangulations, from: "Geometric topology (Proc. Georgia Topology Conf.)", (J C Cantrell, editor), Academic Press, New York (1979) 345-350 MR537740

[11] D E Galewski, R J Stern, Classification of simplicial triangulations of topological manifolds, Ann. of Math. 111 (1980) 1-34 MR558395

[12] M Gromov, Hyperbolic groups, from: "Essays in group theory", (S M Gersten, editor), Math. Sci. Res. Inst. Publ. 8, Springer, New York (1987) 75-263 MR919829

[13] R C Kirby, L C Siebenmann, Foundational essays on topological manifolds, smoothings, and triangulations, Princeton Univ. Press (1977) MR0645390

[14] C Manolescu, Pin(2)-equivariant Seiberg-Witten Floer homology and the triangulation conjecture arXiv:1303.2354

[15] T Matumoto, Triangulation of manifolds, from: "Algebraic and geometric topology Part 2”, (R J Milgram, editor), Proc. Sympos. Pure Math. 32, Amer. Math. Soc. (1978) 3-6 MR520517 
[16] D V Osin, Relatively hyperbolic groups: intrinsic geometry, algebraic properties, and algorithmic problems, Mem. Amer. Math. Soc. 843, Amer. Math. Soc. (2006) MR2182268

[17] N Saveliev, Invariants for homology 3-spheres, Encyclopaedia of Mathematical Sciences 140, Springer, Berlin (2002) MR1941324

[18] L C Siebenmann, Are nontriangulable manifolds triangulable?, from: "Topology of Manifolds (Proc. Inst., Univ. of Georgia)", (J C Cantrell, C H Edwards, editors), Markham, Chicago, Ill. (1970) 77-84 MR0271956

Department of Mathematics, The Ohio State University 231 West 18th Ave., Columbus, OH 43210-1174, USA

davis.12@math.osu.edu, fowler@math.ohio-state.edu, jlafont@math. ohio-state.edu

http://www.math. osu.edu/ davis.12/,

http://www.math.osu.edu/ fowler.291/,

http://www.math.osu.edu/ lafont.1/

Received: 12 May $2013 \quad$ Revised: 25 August 2013 